\title{
Interplay between magnetism and interface-induced effects in ultrathin manganites
}

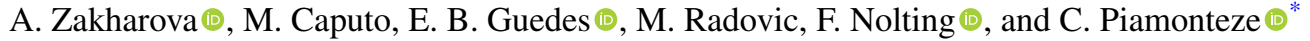 \\ Swiss Light Source, Paul Scherrer Institute, 5232 Villigen PSI, Switzerland
}

(Received 27 July 2021; accepted 8 November 2021; published 3 December 2021)

\begin{abstract}
Optimally doped manganite $\left[\mathrm{La}_{0.67} \mathrm{Sr}_{0.33} \mathrm{MnO}_{3}\right.$ (LSMO)] is a ferromagnetic material with perspective use in applications due to colossal magnetoresistance and $T_{c}$ around room temperature. However, utilizing LSMO in thin-film form and complex heterostructures is limited due to the appearance of a magnetic dead layer, but interfacing LSMO with other transition metal oxides (TMOs) can reduce the magnetic dead layer. For instance, the LSMO layer in proximity to $\mathrm{SrRuO}_{3}$ (SRO) remains magnetic down to 1-2 u.c. To illuminate this effect, we study the magnetic properties and orbital anisotropy of ultrathin LSMO deposited on $\mathrm{SrTiO}_{3}$ (STO) compared with and without an SRO intermediate layer by resonant x-ray spectroscopy. We found that two events occur at the LSMO-SRO interface: Orbital rearrangement and charge transfer. Both effects cooperate, enhancing in-plane double exchange in ultrathin LSMO. Based on quantitative analysis and theoretical simulation of the $\mathrm{x}$-ray spectra, magnetic stability mechanisms in LSMO/SRO are discussed in detail.
\end{abstract}

DOI: 10.1103/PhysRevMaterials.5.124404

\section{INTRODUCTION}

Transition metal oxides (TMOs) have been an attractive area of research and widely studied for the last decades. Perovskite TMOs possess very complex and fascinating properties due to strong electronic correlation. Moreover, dimensionality, strain, and proximity effects induce extraordinary properties in TMO thin films and heterostructures, which differ from the bulk materials [1]. Perovskite manganite $\mathrm{La}_{0.67} \mathrm{Sr}_{0.33} \mathrm{MnO}_{3}$ (LSMO) is one of the TMOs showing the highest Curie temperature $\left(T_{c}=369 \mathrm{~K}\right)$ among manganites in its bulk form and became famous due to its colossal magnetoresistance effect [1-4]. Below the transition temperature, it is a half-metallic ferromagnet demonstrating the remarkable potential for magnetic memory device applications [5]. In LSMO, Mn sits in an octahedral site surrounded by six oxygen atoms $\mathrm{MnO}_{6}$, and the ferromagnetism is mediated through double exchange (DE) between $\mathrm{Mn}^{3+}$ and $\mathrm{Mn}^{4+}$ ions through oxygen [6]. While $\mathrm{Mn}^{3+}$ has one electron in the $e_{g}$ orbital, $\mathrm{Mn}^{4+}$ has a symmetric charge distribution with the $t_{2 g}$ band half-full. The degeneracy of the $e_{g}$ orbital in $\mathrm{Mn}^{3+}$ can be broken by the Jahn-Teller effect or by strain, resulting in different electronic occupation of $x^{2}-y^{2}$ and $3 z^{2}-r^{2}$ orbitals. The interplay between spin and orbital degrees of freedom causes diverse magnetic behavior of LSMO and raises possibilities of its manipulation [6-8].

In the ultrathin films case, it was reported that the magnetic and electronic properties of LSMO are dramatically affected [9-12]. This effect in LSMO film is attributed to a so-called magnetic dead layer with critical thickness of 5-7 u.c. on $\mathrm{SrTiO}_{3}$ (STO) [13-15]. Different possible explanations for the absence of magnetism have been reported as orbital modification at the interface and surface [16-20], octahedral

\footnotetext{
*cinthia.piamonteze@psi.ch
}

rotation [21,22], and charge redistribution [23]. X-ray linear dichroism (XLD) has been widely used to identify the impact of orbital occupation and orbital modification on magnetism in ultrathin LSMO films [19-24].

Nonetheless, it has been found experimentally that the magnetic dead layer thickness in LSMO thin films interfaced with $\mathrm{SrRuO}_{3}$ (SRO) is reduced [25-28]. SRO is an itinerant ferromagnetic material (with $T_{c}=150 \mathrm{~K}$ ) where, due to the extended nature of $4 d$ orbitals, the superexchange (SE) coupling occurs between $\mathrm{Ru} 4 d$ and $\mathrm{O} 2 p$. The SRO film also exhibits a critical thickness of 5 u.c. below which it turns into a nonmagnetic insulator [29]. Thick SRO film deposited on STO (001) has an out-of-plane magnetic easy axis [30], while for LSMO on STO, it is in plane [31]. In LSMO/SRO heterostructures, $\mathrm{Ru} 4 d$ couples with $\mathrm{Mn} 3 d$ via interfacial oxygen atoms, resulting in antiferromagnetic (AFM) coupling between two ferromagnets $[32,33]$. The overlap between transition metal $d$ and $\mathrm{O} 2 p$ orbitals directly influences the strength and sign of the indirect magnetic exchange interaction; therefore, parameters such as orbital occupancy play a key role [6-8]. Lv et al. [34] have performed density functional theory (DFT) calculations showing that LSMO in contact with SRO undergoes an orbital restructuring which, in conjunction with charge transfer, stabilizes a robust ferromagnetism at LSMO below its critical thickness. However, currently, no experimental evidence for such orbital reconstruction is presented.

To understand the influence of SRO on the orbital reconstruction and charge transfer and linking it to the LSMO magnetic properties, we investigate here LSMO/SRO bilayers deposited on STO in comparison with LSMO thin film grown on STO substrate by means of polarized soft x-ray absorption. By employing x-ray magnetic circular dichroism (XMCD), we confirm that the magnetic dead layer is strongly reduced or absent when the SRO layer is inserted between LSMO and STO. XLD results show an orbital reconstruction for 


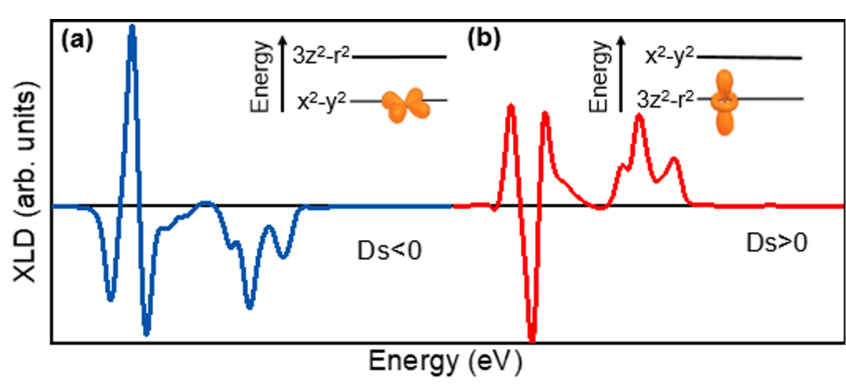

FIG. 1. Ligand field multiplet simulations of x-ray linear dichroism (XLD) spectra for $\mathrm{Mn}^{3+}$. Parameters are given in Table I, where a single $e_{g}$ electron occupies (a) the $x^{2}-y^{2}$ orbital or (b) the $3 z^{2}-r^{2}$ orbital.

LSMO/SRO. However, in the ultrathin limit, the orbital occupation is similar for all films regardless of the interface. We discuss how this apparent similarity leads to contrasting magnetic properties. In addition, x-ray absorption spectroscopy (XAS) shows that the Mn mixed valence in LSMO is preserved throughout the film thickness when interfaced with $\mathrm{SRO}$, in contrast to a $\mathrm{Mn}^{3+}$ accumulation in the interface with STO.

\section{EXPERIMENT DETAILS}

Epitaxial heterostructures of LSMO/SRO were deposited on $\mathrm{TiO}_{2}$-terminated STO (001) by pulsed laser deposition using stoichiometric targets of LSMO (La 66\%, Sr 33\%) and SRO. The fourth harmonic of a Nd:YAG laser at a repetition rate of $2 \mathrm{~Hz}$ was used to ablate the target while keeping the substrate at a temperature of $720 \mathrm{~K}$ in an oxygen atmosphere of $0.1 \mathrm{mbar}$. The thickness and quality control of films were monitored in situ during the growth using reflection highenergy electron diffraction. LSMO films of 2, 4, 8, and 15 u.c. were deposited on 20 u.c. SRO and are labeled from now on as $\frac{2}{20}, \frac{4}{20}, \frac{8}{20}$, and $\frac{15}{20}$, respectively. For comparison, a 4 -u.c.thick LSMO layer was deposited on nonferromagnetic 3 u.c. SRO $\left(\frac{4}{3}\right)$. In addition, single layers of LSMO films of varying thicknesses were deposited directly on STO. The single-layer LSMO films are labeled as $\frac{4}{0}, \frac{8}{0}, \frac{11}{0}$, and $\frac{25}{0}$, where the numerators correspond to the LSMO thickness in unit cells. One unit cell of LSMO and SRO corresponds to $\sim 0.39 \mathrm{~nm}$ [31]. Structural characterization of the heterostructures was done by $\mathrm{x}$-ray diffraction (XRD) and $\mathrm{x}$-ray reflectivity (XRR) measurements using a Bruker D8 Discover diffractometer.

The Mn $L_{2,3}$-edge absorption spectra were obtained at the EPFL/PSI X-Treme beamline at the Swiss Light Source [35] in total electron yield (TEY) mode. The XAS is defined as the sum of the spectra measured with different polarizations. The XMCD and XLD were normalized such that the corresponding maximum XAS was at 1 . Spectra were measured in grazing incidence geometry with a $30^{\circ}$ angle between the sample plane and incoming $\mathrm{x}$ rays. The XMCD spectra was calculated as the difference between XAS measured with circularly polarized right and left $\mathrm{x}$ rays. The XMCD were recorded with applied field of $6 \mathrm{~T}$ or with $50 \mathrm{mT}$ after saturation at $6 \mathrm{~T}$. The magnetic field was applied parallel to the X-ray beam. All temperature-dependent XMCD data were measured after zero-field cooling. Sum rules were applied to
TABLE I. CTM4XAS parameters used for calculation in Fig. 1.

\begin{tabular}{lcrrrc}
\hline \hline$e_{g}$ occupancy & $10 D_{q}$ & \multicolumn{1}{c}{$D_{t}$} & \multicolumn{1}{c}{$D_{s}$} & $\Delta$ & $U_{p d}-U_{d d}$ \\
\hline$x^{2}-y^{2}$ & 1.7 & -0.01 & -0.16 & 4 & 2.5 \\
$3 z^{2}-r^{2}$ & 1.7 & 0.01 & 0.16 & 4 & 2.5 \\
\hline \hline
\end{tabular}

XMCD spectra using the following equations for orbital and spin magnetic moments [36-38]:

$$
\begin{aligned}
m_{\mathrm{orb}}= & \frac{4 \int_{L_{3}+L_{2}}\left(\mu_{+}-\mu_{-}\right) d \omega}{3 \int_{L_{3}+L_{2}}\left(\mu_{+}+\mu_{-}\right) d \omega}\left(10-n_{3 d}\right), \\
m_{\mathrm{spin}}= & \frac{6 \int_{L_{3}}\left(\mu_{+}-\mu_{-}\right) d \omega-4 \int_{L_{3}+L_{2}}\left(\mu_{+}-\mu_{-}\right) d \omega}{\int_{L_{3}+L_{2}}\left(\mu_{+}+\mu_{-}\right) d \omega} \\
& \times\left(10-n_{3 d}\right),
\end{aligned}
$$

where $10-n_{3 d}$ is the number of Mn holes, and it was taken as 5.5 from Ref. [39]. At the end, the spin moment value was corrected by a factor of 1.5 due to admixture of $\mathrm{Mn} L_{2}$ and $L_{3}$ edges [40,41].

The XLD spectra were calculated as the intensity difference between the spectra measured with in-plane $\mathbf{E} \| a b\left(I_{a b}\right)$ and out-of-plane $\mathbf{E} \| c\left(I_{c}\right)$ polarizations probing the $e_{g} x^{2}-y^{2}$ and $3 z^{2}-r^{2}$ orbitals, i.e., $I_{a b}-I_{c}$. The measurements were done with $\pi$ and $\sigma$ polarization. While the measurement with $\sigma$ polarization corresponds directly to $I_{a b}$, the $\pi$ polarization measurement contains both in-plane and out-of-plane components related by the incidence angle. The actual $I_{c}$ absorption for $30^{\circ}$ grazing can be obtained by

$$
I_{c}=\frac{I_{\pi}-I_{\sigma} \sin ^{2} 30^{\circ}}{\cos ^{2} 30^{\circ}}=\frac{4}{3}\left(I_{\pi}-\frac{1}{4} I_{\sigma}\right) .
$$

For a quantitative analysis of the experimental XLD, the hole ratio of $e_{g}$ orbitals was calculated as [42]

$$
X=\frac{h\left(3 z^{2}-r^{2}\right)}{h\left(x^{2}-y^{2}\right)}=\frac{3 I_{c}}{4 I_{a b}-I_{c}} .
$$

The error bars for $X$ were calculated as the standard deviation between different XLD measurements carried out at three different spots of the samples.

There were two basic ligand field multiplet simulations of XLD performed using CTM4XAS [43], shown in Fig. 1 for the single $\mathrm{Mn}^{3+} e_{g}$ electron occupying either the $3 d\left(x^{2}-y^{2}\right)$ orbital [Fig. 1(a)] or the $3 d\left(3 z^{2}-r^{2}\right)$ orbital [Fig. 1(b)]. The simulations are for $\mathrm{Mn}^{3+}$ only since $\mathrm{Mn}^{4+}$ does not exhibit linear dichroism. The simulations were done in $D 4_{h}$ symmetry with charge transfer using the parameters shown in Table I. In this table, $10 D_{q}$ corresponds to the cubic crystal field splitting, while $D_{t}$ and $D_{s}$ give the tetragonal distortion. The energy splitting of the $t_{2 g}$ and $e_{g}$ levels are given by $3 D_{s}-5 D_{t}$ and $4 D_{s}+5 D_{t}$, respectively. The $3 z^{2}-r^{2}\left(x^{2}-y^{2}\right)$ preferential orbital occupation is induced by positive (negative) sign of $D_{s}$ and $D_{t}$. Here, $\Delta$ gives the energy difference between the nominal state $d^{4}$ and the charge transfer state $d^{5} L$, where $L$ corresponds to a hole in the O $2 p$ level. The dependence on LSMO thickness shown in Fig. 6(b) was obtained by linear combination 


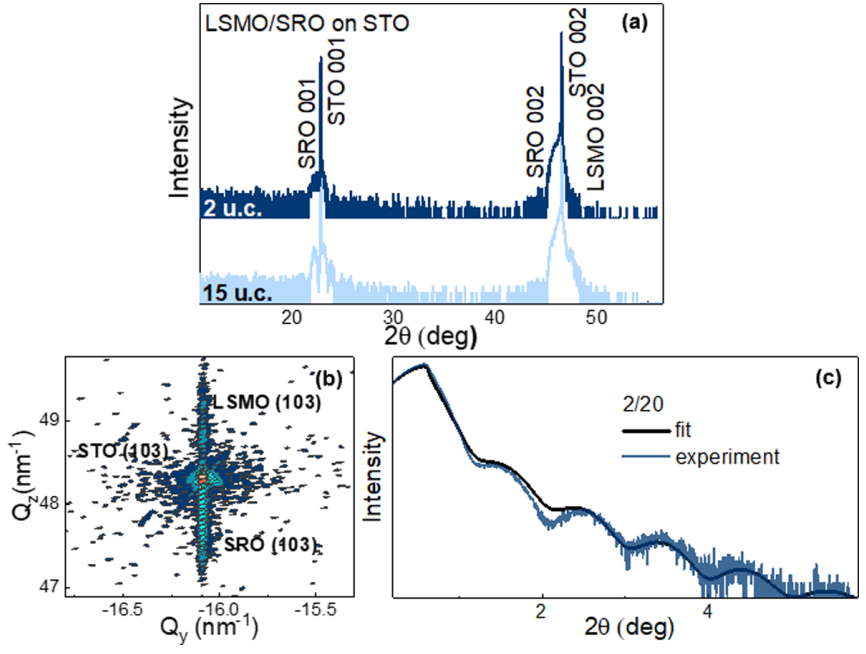

FIG. 2. (a) X-ray diffraction (XRD) $\omega-2 \theta$ scans for $\frac{2}{20}$ and $\frac{15}{20}$. (b) XRD reciprocal space map along $\mathrm{SrTiO}_{3}$ (STO) (103) of $\frac{15}{20}$. (c) $\mathrm{X}$-ray reflectivity (XRR) data and simulated curve for $\frac{2}{20}$.

of the simulations shown in Fig. 1, where the sum of scale factors $=1$

\section{RESULTS}

\section{A. Structural characterization}

The pseudocubic lattice parameter for LSMO is $a_{\mathrm{LSMO}}=$ $3.87 \AA$, while for STO, it is $a_{\mathrm{STO}}=3.905 \AA$ and $a_{\mathrm{SRO}}=$ $3.93 \AA$ for SRO. Figure 2 (a) shows a XRD $\omega-2 \vartheta$ scan along the (001) reflection of STO for $\frac{2}{20}$ and $\frac{15}{20}$. The strong and sharp substrate peak is observed for both samples with LSMO and SRO film peaks appearing at higher and lower $2 \theta$ values, respectively, as expected from the strain state: tensile for the LSMO layer and compressive for SRO. The thin films have a broader peak than the substrate due to the reduced thickness.

To confirm the growth of fully strained film, we obtained the reciprocal space map around (103), which is shown in Fig. 2(b). LSMO and SRO peaks are located at higher and lower $Q_{z}$ than the substrate peak, respectively, in agreement with Fig. 2(a). All peaks fall at the same $Q_{y}$ value, indicating perfect in-plane matching and confirming that both layers are fully strained.

As an indication of the high-quality of the interface, in Fig. 2(c), the black curve presents the Kiessig fringe pattern on the $\frac{2}{20}$ sample. The thickness determination of each layer based on fitting of reflectivity curves [Fig. 2(c), blue curve] agrees with the expected values, and a roughness of $0.1-$ $0.2 \mathrm{~nm}$ was obtained for each layer.

\section{B. Magnetic properties}

To capture the $T_{c}$ of the ultrathin LSMO layers, XMCD spectra were measured at the $\mathrm{Mn} L_{2,3}$ edge, for both bilayers and single layers, at different temperatures, with an applied field of $50 \mathrm{mT}$. Here, $50 \mathrm{mT}$ field was chosen to avoid measuring an "unknown" field since it is not possible to have absolutely zero in a superconducting magnet. The XMCD intensity difference, defined as the difference
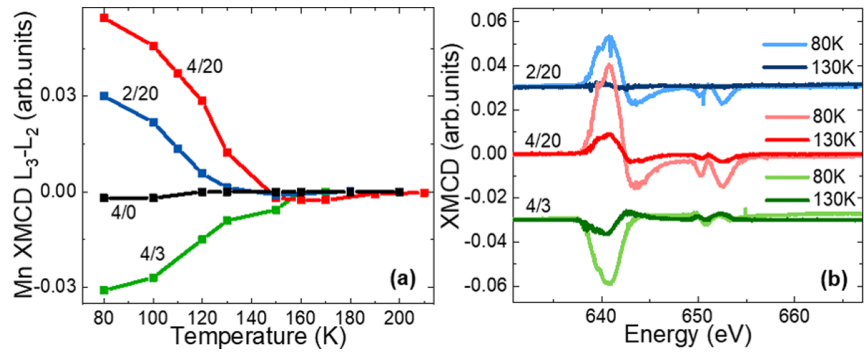

FIG. 3. (a) Difference between Mn x-ray magnetic circular dichroism (XMCD) intensity at $L_{3}$ and $L_{2}$ edges plotted as a function of temperature. (b) $\mathrm{Mn} L_{2,3} \mathrm{XMCD}$ comparison at two temperatures: 80 and $130 \mathrm{~K}$. Data in (a) and (b) were obtained in grazing incidence geometry at $50 \mathrm{mT}$ applied after saturation for ultrathin $\mathrm{La}_{0.67} \mathrm{Sr}_{0.33} \mathrm{MnO}_{3}$ (LSMO; 2 and 4 u.c. thick) for three different interface layers $(0,3$, and 20 u.c. thick).

between its maxima/minima at the $L_{3}$ and $L_{2}$ edges $\left(L_{3}-L_{2}\right)$, is plotted in Fig. 3(a) as a function of temperature. As expected, $\frac{4}{0}$ shows no measurable magnetic remanence down to $80 \mathrm{~K}$, while all other bilayers show magnetic remanence, even the $\frac{2}{20}$, which has an LSMO thickness much below the single film critical thickness. The temperatures at which XMCD response vanishes in Fig. 3(a) were found as $130 \mathrm{~K}$ for $\frac{2}{20}$ and $150 \mathrm{~K}$ for $\frac{4}{20}$ and $\frac{4}{3}$. In Fig. 3(b), comparison of XMCD spectra measured at 80 with $130 \mathrm{~K}$ confirms observation from Fig. 3(a) that the $\frac{2}{20}$ has no significant remanence at $130 \mathrm{~K}$, while 4 u.c. LSMO on SRO is still magnetic, and the absolute value of the remanent moment is the same for $\frac{4}{3}$ and $\frac{4}{20}$. Therefore, $T_{c}$ values that were found for the bilayers are indeed lower than $T_{c}$ in LSMO bulk, but they are clearly higher than $T_{c}$ of thin LSMO single film of similar thicknesses, as previously observed [10]. Thus, we can conclude that LSMO magnetism recovers in the ultrathin limit when interfaced with an SRO layer. This recovery happens even for films on 3 u.c. SRO, where SRO is not ferromagnetic.

Interestingly, the Mn XMCD signal in Figs. 3(a) and 3(b) is opposite for $\frac{4}{3}$ compared with $\frac{2}{20}$ and $\frac{4}{20}$. In our geometry, this indicates that the $\mathrm{Mn}$ moment projection along the $\mathrm{x}$ rays is oriented opposite to the field direction for $\frac{2}{20}$ and $\frac{4}{20}$, whereas $\frac{4}{3}$ is along the field. This sign flip is a direct observation of the AFM coupling between LSMO and SRO according to the Goodenough-Kanamori rule [32]. Theoretical calculation showed that this condition is energetically more favorable than any other $[33,34]$. At $\frac{4}{3}$, the Mn moment is parallel to the field since 3 u.c. SRO is expected to be nonmagnetic [29].

Next, we look at the magnetic properties of the bilayers for varying LSMO thicknesses while fixing the SRO layer to 20 u.c thick. The XMCD spectra measured at $25 \mathrm{~K}, 50 \mathrm{mT}$ are presented in Fig. 4(a), and the Mn hysteresis curves, measured by XMCD, are presented in Fig. 4(b). Das et al. [44] have also reported the hysteresis of LSMO/SRO bilayers probed by XMCD, for thicker LSMO layers, showing similar shapes as presented here. The data shown in Fig. 4 can be understood looking them as two subdatasets: the thin LSMO layers $\left(\frac{2}{20}\right.$ and $\frac{4}{20}$ ) and the thicker LSMO layers $\left(\frac{8}{20}\right.$ and $\left.\frac{15}{20}\right)$. For the thin layers, the sign of the XMCD at $50 \mathrm{mT}$ [Fig. 4(a)] evidences that the Mn moment is opposite to the applied field, as already 

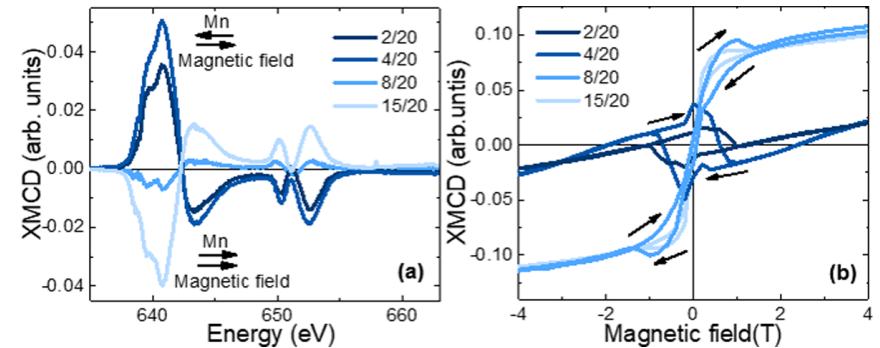

FIG. 4. (a) Comparison of $\mathrm{Mn} L_{2,3}$ x-ray magnetic circular dichroism (XMCD) data measured at $25 \mathrm{~K}$ in grazing in $50 \mathrm{mT}$ after $6 \mathrm{~T}$ between $\mathrm{La}_{0.67} \mathrm{Sr}_{0.33} \mathrm{MnO}_{3}$ (LSMO)/20 $\mathrm{SrRuO}_{3}$ (SRO) bilayers with different LSMO thickness followed by (b) magnetic hysteresis at the $\mathrm{Mn} L_{3}$ edge measured in grazing incidence geometry. Arrows indicate direction of measurements.

observed in Fig. 3. Above $1 \mathrm{~T}\left(\frac{2}{20}\right)$ and $2.5 \mathrm{~T}\left(\frac{4}{20}\right)$, the magnetic field is strong enough to rotate the Mn moments leading to a projection parallel to the applied field, overcoming the AFM coupling of Mn-Ru [Fig. 4(b)]. For the thicker LSMO layers $\left(\frac{8}{20}\right.$ and $\left.\frac{15}{20}\right)$, the Mn moment projection is parallel to the x-ray beam and to the applied magnetic field for fields $>50 \mathrm{mT}$, as evidenced both by Figs. 4(a) and 4(b). In addition, it can be seen from Fig. 4(b) that the thicker LSMO layers are not completely saturated, possibly because of the AFM coupling of the interface layers with SRO.

The Mn total magnetic moment was calculated using Eq. (1) as the sum of spin and orbital contribution. The results are shown in Fig. 5. The Mn saturated moment for $\frac{15}{20}$ in Fig. 5 has been found to be $2.4 \mu_{\mathrm{B}}$, which is less than the expected value of $3.5 \mu_{\mathrm{B}}$ [14]. However, the saturated $\mathrm{Mn}$ moment found for $\frac{15}{3}$ is $3.1 \mu_{\mathrm{B}}$, which is larger than for $\frac{15}{20}$. The moments in low field are also larger for the layer interfaced with 3SRO than with 20SRO, as can be seen in Fig. 5. The explanation for this difference is that the interfacial layer of LSMO is always driven antiparallel to the applied field by coupling with 20 SRO in our films regardless of LSMO

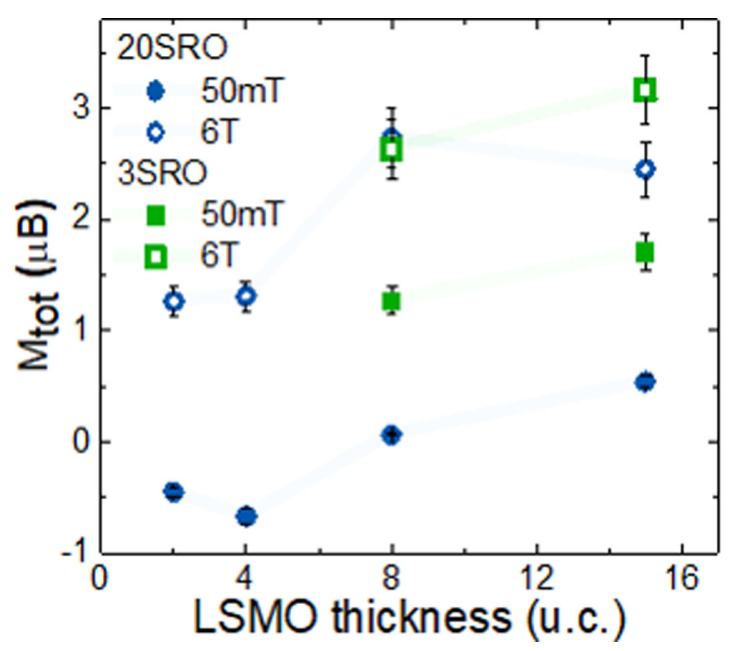

FIG. 5. Total magnetic moment $M_{\text {tot }}$ per Mn atom calculated as a sum of magnetic spin and orbital moment extracted from corresponding $\mathrm{x}$-ray magnetic circular dichroism (XMCD) data at $25 \mathrm{~K}$ in $6 \mathrm{~T}$ and $50 \mathrm{mT}$ using Eq. (1).
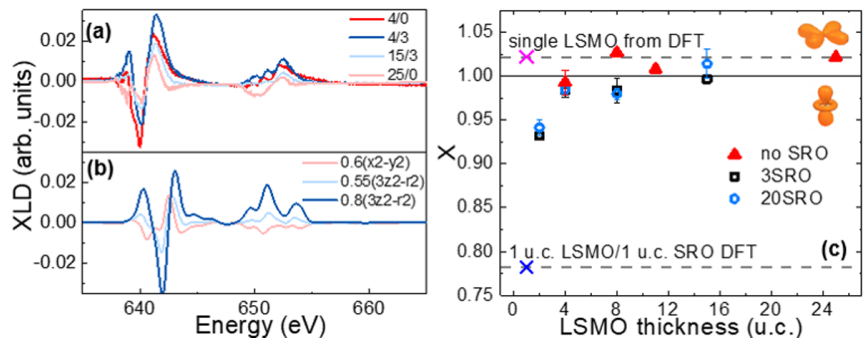

FIG. 6. (a) X-ray linear dichroism (XLD) data collected at Mn $L_{2,3}$ edges in grazing at room temperature. (b) Corresponding linear combination of two simulations labeled as weight of the $3 z^{2}-r^{2}$ orbital. (c) Calculated hole ratio $X$ as a function of $\mathrm{La}_{0.67} \mathrm{Sr}_{0.33} \mathrm{MnO}_{3}$ (LSMO) thickness calculated from experimental XLD data and from density functional theory (DFT) calculation from Ref. [34].

thickness [44]. Top layers of LSMO on 20SRO for thicker LSMO layers tend to align with the field, resulting in the parallel moment projection observed in Fig. 4(b). Since 3 u.c. of SRO is nonmagnetic, the AFM coupling at the interface does not take place, and the moments of LSMO on 3SRO are larger.

\section{Orbital occupation}

Lv et al. [34] have performed DFT calculations showing an orbital reoccupation in $\mathrm{Mn}$ at $\mathrm{LSMO} / \mathrm{SRO}$ in comparison with single LSMO on STO. To validate these calculations, we have investigated the orbital occupation using XLD at Mn $L_{2,3}$ edges [Fig. 6(a)] comparing two different bilayers as a function of LSMO thickness. In LSMO, $\mathrm{Mn}^{4+}$ does not provide any XLD signal since the $e_{g}$ orbital is empty and $t_{2 g}$ is half-full. Therefore, the XLD signal represents the anisotropy of $e_{g}$ orbital in $\mathrm{Mn}^{3+}$. The spectra in Fig. 6(a) are not well reproduced by the simulations for single orbital occupation presented in Figs. 1(a) and 1(b). This is because both orbitals are partially occupied. Therefore, we simulated the spectra by making a linear combination of the simulations in Fig. 1, which is shown in Fig. 6(b). The differences among the XLD spectra measured for the various bilayers are subtle. In the following discussion, we focus on the differences at the $L_{2}$ edge since the XLD spectra is less complex. As seen in Fig. 1, a single electron at $x^{2}-y^{2}$ would give a negative XLD at the $L_{2}$ edge, while $3 z^{2}-r^{2}$ occupation contributes to a positive XLD at the $L_{2}$ edge. We start by discussing the thickest single layer measured $\frac{25}{0}$. For this film, the XLD shows a down-up feature, which agrees with the simulation shown in Fig. 6(b), having the 0.6 weight from the $x^{2}-y^{2}$ orbital occupied. For three other films presented $\left(\frac{4}{3}, \frac{15}{3}\right.$, and $\left.\frac{4}{0}\right)$, the $L_{2}$ XLD is positive, which agrees better with the simulations where $3 z^{2}-r^{2}$ is occupied in majority.

For a quantitative interpretation of the XLD spectra, we have calculated the ratio $X$ between holes at the two $e_{g}$ orbitals $h\left(3 z^{2}-r^{2}\right) / h\left(x^{2}-y^{2}\right)$, as given in Eq. (3). The values obtained for $X$ are plotted in Fig. 6(c) as a function of LSMO thickness. Here, $X>1(<1)$ indicates that in-plane (out-of-plane) orbitals have higher occupation. The simulation from Fig. 6(b) agrees with the experimental data in Fig. 6(c), which show 


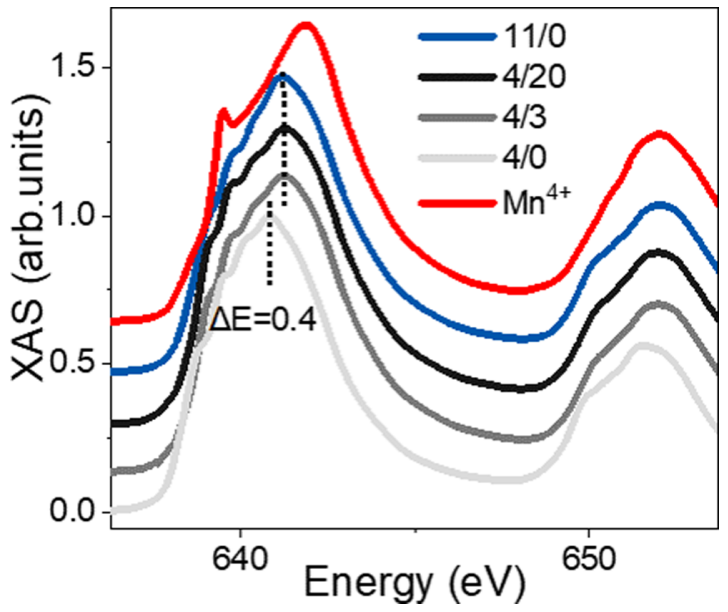

FIG. 7. Comparison of x-ray absorption spectroscopy (XAS) at Mn $L_{2,3}$ edges measured at $300 \mathrm{~K}$ between 4 u.c. $\mathrm{La}_{0.67} \mathrm{Sr}_{0.33} \mathrm{MnO}_{3}$ (LSMO) interfaced with different $\mathrm{SrRuO}_{3}$ (SRO) layers $(0,3$, and 20 u.c. thick) and reference data $\left(\mathrm{SrMnO}_{3}\right.$ and $\left.\frac{11}{0}\right)$.

the $3 z^{2}-r^{2}\left(x^{2}-y^{2}\right)$ orbital more occupied, indeed also show $X<1(>1)$.

From Fig. 6(b), we see that there is a change of orbital occupation with thickness: 4- and 2-u.c.-thick LSMO films have out-of-plane orbital ordering, while thicker films have in-plane orbital ordering. Since LSMO is tensile strained on STO substrate, from a pure strain point of view, it is expected that the orbital occupancy should be dominantly from $x^{2}-y^{2}$ orbitals. Indeed, the $X$ value found for $\frac{25}{0}$ matches with this prediction, and it is in very good agreement with theoretical DFT calculation for single LSMO film on STO. For thinner LSMO layers $(<8$ u.c.) deposited on SRO, we find a change of orbital contribution with a larger $3 z^{2}-r^{2}$ occupation for LSMO, also in agreement with the published calculations [34]. However, the calculated values give $\sim 15-20 \%$ larger orbital symmetry for 1 u.c. LSMO on SRO, than what we measure for 2 u.c. of LSMO on SRO.

In contrast to thick LSMO/STO films (LSMO $>4$ u.c.), the XLD shape of $\frac{4}{0}$ and the calculated $X$ show out-of-plane occupation, very similar to $\frac{4}{3}$ [Figs. 6(a) and 6(b)]. This agrees with previous observations of ultrathin single LSMO that demonstrate orbital occupancy opposite to the one expected due to pure strain $[20,21,24]$.

\section{Charge transfer}

Finally, we have also looked at the Mn valence for bilayers compared with single layers by comparing the XAS at Mn $L_{2,3}$ edges. Figure 7 shows XAS spectra for $\frac{11}{0}, \frac{4}{0}$, and $\frac{4}{20}$. The XAS from powder $\mathrm{SrMnO}_{3}$ is also shown as a reference for $\mathrm{Mn}^{4+}$. From Fig. 7, $\frac{11}{0}, \frac{4}{20}$, and $\frac{4}{3}$ all have maximum XAS peaks at a similar energy. The energy position of XAS peaks is lower than in $\mathrm{Mn}^{4+}$ of the reference sample, which is expected due to the mixed valence of LSMO [45]. On the other hand, $\frac{4}{0}$ has a clear energy shift of $\sim 0.4 \mathrm{eV}$ toward lower energy, evidencing a lower Mn valence state in $\frac{4}{0}$ than the other films.

Here, we point out that the TEY signal has exponential decay on the electron escape depth. Then consequently, relative contribution of the buried layers to the total signal decreases as the film thickness increases. Therefore, it is reasonable to assume that the energy shift between $\frac{11}{0}$ and $\frac{4}{0}$ is due to a higher $\mathrm{Mn}^{3+}$ concentration at the LSMO/STO interface. This valence change with thickness is in agreement with previous results $[15,23,46,47]$.

Since the 4-u.c.-thick LSMO layer interfaced with the SRO layer (20 and 3 u.c.) does not exhibit an energy shift compared with $\frac{11}{0}$, we conclude that the nominal Mn mixed valence in LSMO is preserved through the entire thickness in the LSMO/SRO bilayer. Such a valence stability could be evoked by increased charge transfer at the LSMO/SRO interface. It is expected that, due to the extended nature of the $\mathrm{Ru} 4 d$ orbital, $\mathrm{O} 2 p$ hybridizes more with $\mathrm{Ru} 4 d$ than with $\mathrm{Ti} 3 d$ [48]. At the same time, SRO is in the negative charge transfer regime, meaning that $\mathrm{Ru} d^{4}$ is more likely to accept an electron from O $2 p$ rather than transferring its own electrons [49].

\section{DISCUSSION}

Our XMCD measurements shown in Sec. III B confirm that the LSMO layer is magnetically dead for the thickness of 4 u.c. when grown on STO. However, once the ultrathin LSMO layer is interfaced with the 20 u.c. SRO layer $\left(\frac{4}{20}\right.$ and even $\frac{2}{20}$ ), the magnetic order is restored, indicating that the dead layer is reduced dramatically. It is important to notice that, even when the SRO layer is not ferromagnetic, the LSMO ferromagnetism is still preserved, as in $\frac{4}{3}$. In addition, our results suggest a change in LSMO moment orientation throughout the layer thickness with the moments close to the LSMO/SRO interface antiparallel to the applied field and the top layers parallel to the applied field.

Our films are completely strained, as shown in Fig. 2, and indeed, the orbital anisotropy measured for $\frac{11}{0}$ agrees with LSMO in tensile strain (Fig. 6). The interface with SRO leads to out-of-plane orbital occupation, in qualitative agreement with DFT predictions [34]. However, 4 u.c. LSMO on STO also shows out-of-plane orbital occupation, in agreement with previously reported studies $[20,21,24]$. In summary, $\frac{4}{0}, \frac{4}{3}$, and $\frac{4}{20}$ show very similar orbital occupation despite their contrastingly different magnetic properties. We discuss in sequence how this apparently contradicting result can be reconciled.

The out-of-plane orbital occupation in ultrathin LSMO on STO has been previously observed and associated with Ctype AFM ordering, where the crystal field splitting of the $e_{g}$ levels lowers the $\left(3 z^{2}-r^{2}\right)$ orbitals [20]. In addition, the larger $\mathrm{Mn}^{3+}$ concentration observed at the interface would result in disrupted FM ordering in plane and loss of metallicity.

Here, SRO on STO grows on compressive strain, and the energetic levels are such that $\mathrm{Ru} t_{2 g} x z$ and $y z$ orbitals are lower in energy [29]. Since in-plane overlap between Mn $3 d$ and Ru $4 d$ is weak, only out-of-plane orbitals can contribute to $\mathrm{Mn}$ $\mathrm{Ru}$ coupling. In LSMO/SRO, this orbital overlap allows for a three-dimensional electronic structure, hindering quantum confinement effects [25]. The hybridized Mn $3 d 3 z^{2}-r^{2}$ orbital drops below the $x^{2}-y^{2}$, changing the sign of LSMO XLD, as observed here. Furthermore, the LSMO/SRO interface hinders the Mn valence reduction observed at the LSMO/STO interface, and the nominal LSMO valence is preserved throughout 
the film thickness. In summary, the hybridization with the $\mathrm{Ru}$ orbitals coming from the orbital reconstruction, together with the preservation of the Mn valence, help in reducing the LSMO magnetic dead layer. In addition, the remaining Mn $x^{2}-y^{2}$ occupation still allows for a strong Mn DE in plane.

\section{CONCLUSIONS}

We have revealed the large impact of proximity and dimensionality, resulting in the magnetic dead layer in LSMO/SRO bilayers being drastically diminished. This restoration of magnetism in the ultrathin LSMO layer could be associated with strong hybridization between LSMO and SRO, hindering the quantum confinement effect. Even though the Mn orbital occupation in ultrathin layers probed by XLD is the same, this orbital rearrangement has different origins and consequences.
In LSMO/SRO bilayers, it allows for a strong hybridization at the LSMO/SRO interface, leading to an effective $\mathrm{Mn}-\mathrm{Ru}$ coupling. Additionally, in LSMO/SRO bilayers, in contrast to LSMO single layers, the Mn valence is maintained throughout the thickness, allowing for the preservation of the DE coupling in plane.

\section{ACKNOWLEDGMENTS}

A.Z. acknowledges the financial support by the Swiss National Science Foundation (SNSF) under Project No. 200021_169467. M.R. and E.B.G. acknowledge SNF support, Project No. 200021_182695. A.Z. thanks Laura Maurel for the help with the diffraction measurements. Part of the work was carried out at the EPFL/PSI X-Treme beamline at Swiss Light Source, Paul Scherrer Institut, Switzerland.
[1] P. Zubko, S. Gariglio, M. Gabay, P. Ghosez, and J. M. Triscone, Interface physics in complex oxide heterostructures, Annu. Rev. Condens. Matter Phys. 2, 141 (2011).

[2] A. Urushibara, Y. Moritomo, T. Arima, A. Asamitsu, G. Kido, and Y. Tokura, Insulator-metal transition and giant magnetoresistance in $\mathrm{La}_{1-x} \mathrm{Sr}_{x} \mathrm{MnO}_{3}$, Phys. Rev. B 51, 14103 (1995).

[3] Y. Suzuki, H. Y. Hwang, S. W. Cheong, and R. B. van Dover, The role of strain in magnetic anisotropy of manganite thin films, Appl. Phys. Lett. 71, 140 (1997).

[4] H. L. Liu, K. S. Lu, M. X. Kuo, L. Uba, S. Uba, L. M. Wang, and H.-T. Jeng, Magneto-optical properties of $\mathrm{La}_{0.7} \mathrm{Sr}_{0.3} \mathrm{MnO}_{3}$ thin films with perpendicular magnetic anisotropy, J. Appl. Phys. 99, 043908 (2006).

[5] J.-H. Park, E. Vescovo, H.-J. Kim, C. Kwon, R. Ramesh, and T. Venkatesan, Direct evidence for a half-metallic ferromagnet, Nature 392, 794 (1998).

[6] Z. Fang, I. V. Solovyev, and K. Terakura, Phase Diagram of Tetragonal Manganites, Phys. Rev. Lett. 84, 3169 (2000).

[7] Y. Tokura and N. Nagaosa, Orbital physics in transition-metal oxides, Science 288, 462 (2000).

[8] A. Sadoc, B. Mercey, C. Simon, D. Grebille, W. Prellier, and M.-B. Lepetit, Large Increase of the Curie Temperature by Orbital Ordering Control, Phys. Rev. Lett. 104, 046804 (2010).

[9] M. Bibes, L. Balcells, S. Valencia, J. Fontcuberta, M. Wojcik, E. Jedryka, and S. Nadolski, Nanoscale Multiphase Separation at $\mathrm{La}_{2 / 3} \mathrm{Ca}_{1 / 3} \mathrm{MnO}_{3} / \mathrm{SrTiO}_{3}$ Interfaces, Phys. Rev. Lett. 87, 067210 (2001).

[10] M. Huijben, L. W. Martin, Y.-H. Chu, M. B. Holcomb, P. Yu, G. Rijnders, D. H. A. Blank, and R. Ramesh, Critical thickness and orbital ordering in ultrathin $\mathrm{La}_{0.7} \mathrm{Sr}_{0.3} \mathrm{MnO}_{3}$ films, Phys. Rev. B 78, 094413 (2008).

[11] L. Chen, Z. Wang, G. Wang, H. Guo, M. Saghayezhian, Z. Liao, Y. Zhu, E. W. Plummer, and J. Zhang, Surface and interface properties of $\mathrm{La}_{2 / 3} \mathrm{Sr}_{1 / 3} \mathrm{MnO}_{3}$ thin films on $\mathrm{SrTiO}_{3}(001)$, Phys. Rev. Materials 3, 044407 (2019).

[12] M. Angeloni, G. Balestrino, N. G. Boggio, P. G. Medaglia, P. Orgiani, and A. Tebano, Suppression of the metal-insulator transition temperature in thin films, J. Appl. Phys. 96, 6387 (2004).

[13] J. Z. Sun and D. W. Abraham, Thickness-dependent magnetotransport in ultrathin manganite films, Appl. Phys. Lett. 74, 3017 (1999).
[14] H. Boschker, J. Kautz, E. P. Houwman, W. Siemons, D. H. A. Blank, M. Huijben, G. Koster, A. Vailionis, and G. Rijnders, High-Temperature Magnetic Insulating Phase in Ultrathin $\mathrm{La}_{0.67} \mathrm{Sr}_{0.33} \mathrm{MnO}_{3}$ Films, Phys. Rev. Lett. 109, 157207 (2012).

[15] J.-S. Lee, D. A. Arena, P. Yu, C. S. Nelson, R. Fan, C. J. Kinane, S. Langridge, M. D. Rossell, R. Ramesh, and C.-C. Kao, Hidden Magnetic Configuration in Epitaxial $\mathrm{La}_{1-x} \mathrm{Sr}_{x} \mathrm{MnO}_{3}$ Films, Phys. Rev. Lett. 105, 257204 (2010).

[16] N. Mottaghi, M. S. Seehra, R. Trappen, S. Kumari, C. Y. Huang, S. Yousefi, G. B. Cabrera, A. H. Romero, and M. B. Holcomb, Insights into the magnetic dead layer in $\mathrm{La}_{0.7} \mathrm{Sr}_{0.3} \mathrm{MnO}_{3}$ thin films from temperature, magnetic field and thickness dependence of their magnetization, AIP Adv. 8, 056319 (2018).

[17] C. Aruta, G. Ghiringhelli, V. Bisogni, L. Braicovich, N. B Brookes, A. Tebano, and G. Balestrino, Orbital occupation, atomic moments, and magnetic ordering at interfaces of manganite thin films, Phys. Rev. B 80, 014431 (2009).

[18] A. Tebano, A. Orsini, P. G. Medaglia, D. Di Castro, G. Balestrino, B. Freelon, A. Bostwick, Y. J. Chang, G. Gaines, E. Rotenberg, and N. L. Saini, Preferential occupation of interface bands in $\mathrm{La}_{2 / 3} \mathrm{Sr}_{1 / 3} \mathrm{MnO}_{3}$ films as seen via angle-resolved photoemission, Phys. Rev. B 82, 214407 (2010).

[19] S. Valencia, L. Peña, Z. Konstantinovic, L. Balcells, R. Galceran, D. Schmitz, F. Sandiumenge, M. Casanove, and B. Martínez, Intrinsic robust antiferromagnetism at manganite surfaces and interfaces, J. Phys.: Condens. Matter 26, 166001 (2014).

[20] A. Tebano, C. Aruta, S. Sanna, P. G. Medaglia, G. Balestrino, A. A. Sidorenko, R. De Renzi, G. Ghiringhelli, L. Braicovich, V. Bisogni, and N. B. Brookes, Evidence of Orbital Reconstruction at Interfaces in Ultrathin $\mathrm{La}_{0.67} \mathrm{Sr}_{0.33} \mathrm{MnO}_{3}$ Films, Phys. Rev. Lett. 100, 137401 (2008).

[21] Z. L. Liao, N. Gauquelin, R. J. Green, S. Macke, J. Gonnissen, S. Thomas, Z. Zhong, L. Li, L. Si, S. Van Aert, P. Hansmann, K. Held, J. Xia, J. Verbeeck, G. Van Tendeloo, G. A. Sawatzky, G. Koster, M. Huijben, and G. Rijnders, Thickness dependent properties in oxide heterostructures driven by structurally induced metal-oxygen hybridization variations, Adv. Funct. Mater. 27, 1606717 (2017).

[22] X. Li, I. Vrejoiu, M. Ziese, A. Gloter, and P. A. van Aken, Impact of interfacial coupling of oxygen octahedra on 
ferromagnetic order in $\mathrm{La}_{0.7} \mathrm{Sr}_{0.3} \mathrm{MnO}_{3} / \mathrm{SrTiO}_{3}$ heterostructures, Sci. Rep. 7, 40068 (2017).

[23] J.-S. Lee, D. A. Arena, T. S. Santos, C. S. Nelson, S. I. Hyun, J. H. Shim, and C.-C. Kao, Controlling competing interactions at oxide interfaces: enhanced anisotropy in $\mathrm{La}_{0.7} \mathrm{Sr}_{0.3} \mathrm{MnO}_{3}$ films via interface engineering, Phys. Rev. B 85, 235125 (2012).

[24] D. Pesquera, G. Herranz, A. Barla, E. Pellegrin, F. Bondino, E. Magnano, F. Sánchez, and J. Fontcuberta, Surface symmetrybreaking and strain effects on orbital occupancy in transition metal perovskite epitaxial films, Nat. Commun. 3, 1189 (2012).

[25] C. Piamonteze, F. Bern, S. R. Venkata Avula, M. Studniarek, C. Autieri, M. Ziese, and I. Lindfors-Vrejoiu, Ferromagnetic order of ultra-thin $\mathrm{La}_{0.7} \mathrm{Ba}_{0.3} \mathrm{MnO}_{3}$ sandwiched between $\mathrm{SrRuO}_{3}$ layers, Appl. Phys. Lett. 118, 152408 (2021).

[26] M. Ziese, I. Vrejoiu, E. Pippel, P. Esquinazi, D. Hesse, C. Etz, J. Henk, A. Ernst, I. V. Maznichenko, W. Hergert, and I. Mertig, Tailoring Magnetic Interlayer Coupling in $\mathrm{La}_{0.7} \mathrm{Sr}_{0.3} \mathrm{MnO}_{3} / \mathrm{SrRuO}_{3}$ Superlattices, Phys. Rev. Lett. 104, 167203 (2010).

[27] F. Bern, M. Ziese, I. Vrejoiu, X. Li, and P. A. van Aken, Magnetic and magnetotransport properties of ultrathin $\mathrm{La}_{0.7} \mathrm{Ba}_{0.3} \mathrm{MnO}_{3}$ epitaxial films embedded in $\mathrm{SrRuO}_{3}$, New J. Phys. 18, 053021 (2016).

[28] M. Ziese, F. Bern, E. Pippel, D. Hesse, and I. Vrejoiu, Stabilization of ferromagnetic order in $\mathrm{La}_{0.7} \mathrm{Sr}_{0.3} \mathrm{MnO}_{3}-\mathrm{SrRuO}_{3}$ superlattices, Nano Lett. 12, 4276 (2012).

[29] K. Ishigami, K. Yoshimatsu, D. Toyota, M. Takizawa, T. Yoshida, G. Shibata, T. Harano, Y. Takahashi, T. Kadono, V. K. Verma, V. R. Singh, Y. Takeda, T. Okane, Y. Saitoh, H. Yamagami, T. Koide, M. Oshima, H. Kumigashira, and A. Fujimori, Thickness-dependent magnetic properties and straininduced orbital magnetic moment in $\mathrm{SrRuO}_{3}$ thin films, Phys. Rev. B 92, 064402 (2015).

[30] M. Ziese, I. Vrejoiu, and D. Hesse, Structural symmetry and magnetocrystalline anisotropy of $\mathrm{SrRuO}_{3}$ films on $\mathrm{SrTiO}_{3}$, Phys. Rev. B 81, 184418 (2010).

[31] A. Vailionis, H. Boschker, W. Siemons, E. P. Houwman, D. H. A. Blank, G. Rijnders, and G. Koster, Misfit strain accommodation in epitaxial $\mathrm{ABO}_{3}$ perovskites: Lattice rotations and lattice modulations, Phys. Rev. B 83, 064101 (2011).

[32] J. Goodenough, Theory of the role of covalence in the perovskite-type manganites [La, M(II)] $\mathrm{MnO}_{3}$, Phys. Rev. 100, 564 (1955).

[33] Y. Lee, B. Caes, and B. N. Harmon, Role of oxygen $2 p$ states for anti-ferromagnetic interfacial coupling and positive exchange bias of ferromagnetic LSMO/SRO bilayers, J. Alloys Compd. 450, 1 (2008).

[34] K. Lv, H. P. Zhu, W. Q. Zou, F. M. Zhang, and X. $\mathrm{S}$. Wu, Charge transfer and orbital reconstruction in the $\left(\mathrm{La}_{2 / 3} \mathrm{Sr}_{1 / 3} \mathrm{MnO}_{3}\right)_{m} /\left(\mathrm{SrRuO}_{3}\right)_{n}$ superlattices, J. Appl. Phys. 117, 185305 (2015).

[35] C. Piamonteze, U. Flechsig, S. Rusponi, J. Dreiser, J. Heidler, M. Schmidt, R. Wetter, M. Calvi, T. Schmidt, H. Pruchova, J. Krempasky, C. Quitmann, H. Brune, and F. Nolting, X-Treme beamline at SLS: X-ray magnetic circular and linear dichroism at high field and low temperature, J. Synchrotron Rad. 19, 661 (2012).

[36] P. Carra, B. T. Thole, M. Altarelli, and X. Wang, X-Ray Circular Dichroism and Local Magnetic Fields, Phys. Rev. Lett. 70, 694 (1993).
[37] B. Thole, P. Carra, F. Sette, and G. van der Laan, X-ray Circular Dichroism as a Probe of Orbital Magnetization, Phys. Rev. Lett. 68, 1943 (1992).

[38] C. T. Chen, Y. U. Idzerda, H.-J. Lin, N. V. Smith, G. Meigs, E. Chaban, G. H. Ho, E. Pellegrin, and F. Sette, Experimental Confirmation of the X-Ray Magnetic Circular Dichroism Sum Rules for Iron and Cobalt, Phys. Rev. Lett. 75, 152 (1995).

[39] J. Heidler, C. Piamonteze, R. V. Chopdekar, M. A. UribeLaverde, A. Alberca, M. Buzzi, A. Uldry, B. Delley, C. Bernhard, and F. Nolting, Manipulating magnetism in $\mathrm{La}_{0.7} \mathrm{Sr}_{0.3} \mathrm{MnO}_{3}$ via piezostrain, Phys. Rev. B 91, 024406 (2015).

[40] C. Piamonteze, P. Miedema, and F. M. F. de Groot, Accuracy of the spin sum rule in XMCD for the transition-metal $L$ edges from manganese to copper, Phys. Rev. B 80, 184410 (2009).

[41] Y. Teramura, A. Tanaka, B. T. Thole, and T. Jo, Effect of Coulomb Interaction on the $\mathrm{X}$-ray magnetic circular dichroism spin sum rule in $3 d$ transition elements, J. Phys. Soc. Jpn. 65, 1053 (1996).

[42] M. Wu, E. Benckiser, M. W. Haverkort, A. Frano, Y. Lu, U. Nwankwo, S. Brück, P. Audehm, E. Goering, S. Macke, V. Hinkov, P. Wochner, G. Christiani, S. Heinze, G. Logvenov, H.-U. Habermeier, and B. Keimer, Strain and composition dependence of orbital polarization in nickel oxide superlattices, Phys. Rev. B 88, 125124 (2013).

[43] E. Stavitski and F. M. F. de Groot, The CTM4XAS program for EELS and XAS spectral shape analysis of transition metal $L$ edges, Micron 41, 687 (2010).

[44] S. Das, A. D. Rata, I. V. Maznichenko, S. Agrestini, E. Pippel, N. Gauquelin, J. Verbeeck, K. Chen, S. M. Valvidares, H. Babu Vasili, J. Herrero-Martin, E. Pellegrin, K. Nenkov, A. Herklotz, A. Ernst, I. Mertig, Z. Hu, and K. Dörr, Low-field switching of noncollinear spin texture at $\mathrm{La}_{0.7} \mathrm{Sr}_{0.3} \mathrm{MnO}_{3}-\mathrm{SrRuO}_{3}$ interfaces, Phys. Rev. B 99, 024416 (2019).

[45] M. Abbate, F. M. F. de Groot, J. C. Fuggle, A. Fujimori, O. Strebel, F. Lopez, M. Domke, G. Kaindl, G. A. Sawatzky, M. Takano, Y. Takeda, H. Eisaki, and S. Uchida, Controlledvalence properties of $\mathrm{La}_{1-x} \mathrm{Sr}_{x} \mathrm{FeO}_{3}$ and $\mathrm{La}_{1-x} \mathrm{Sr}_{x} \mathrm{MnO}_{3}$ studied by soft-x-ray absorption spectroscopy, Phys. Rev. B 46, 4511 (1992).

[46] M. J. Calderon, L. Brey, and F. Guinea, Surface electronic structure and magnetic properties of doped manganites, Phys. Rev. B 60, 6698 (1999).

[47] R. Herger, P. R. Willmott, C. M. Schlepütz, M. Björck, S. A. Pauli, D. Martoccia, B. D. Patterson, D. Kumah, R. Clarke, Y. Yacoby, and M. Döbeli, Structure determination of monolayerby-monolayer grown $\mathrm{La}_{1-x} \mathrm{Sr}_{x} \mathrm{MnO}_{3}$ thin films and the onset of magnetoresistance, Phys. Rev. B 77, 085401 (2008).

[48] J. Garcia-Barriocanal, J. C. Cezar, F. Y. Bruno, P. Thakur, N. B. Brookes, C. Utfeld, A. Rivera-Calzada, S. R. Giblin, J. W. Taylor, J. A. Duffy, S. B. Dugdale, T. Nakamura, K. Kodama, C. Leon, S. Okamoto, and J. Santamaria, Spin and orbital Ti magnetism at $\mathrm{LaMnO}_{3} / \mathrm{SrTiO}_{3}$ interfaces, Nat. Commun. 1, 82 (2010).

[49] E. B. Guedes, M. Abbate, K. Ishigami, A. Fujimori, K. Yoshimatsu, H. Kumigashira, M. Oshima, F. C. Vicentin, P. T. Fonseca, and R. J. O. Mossanek, Core level and valence band spectroscopy of $\mathrm{SrRuO}_{3}$ : Electron correlation and covalence effects, Phys. Rev. B 86, 235127 (2012). 